\title{
Human Umbilical Cord Mesenchymal Stem Cells: A New Therapeutic Option for Tooth Regeneration
}

\author{
Yuanwei Chen,, ${ }^{1}$ Yongchun Yu, ${ }^{2}$ Lin Chen, ${ }^{1}$ Lanfeng Ye, ${ }^{1}$ Junhui Cui, ${ }^{1}$ Quan Sun, ${ }^{1}$ Kaide Li, \\ Zhiyong $\mathrm{Li}^{3}{ }^{3}$ and Lei Liu ${ }^{1}$ \\ ${ }^{1}$ State Key Laboratory of Oral Diseases, West China Hospital of Stomatology, Sichuan University, Chengdu 610041, China \\ ${ }^{2}$ Department of Stomatology, The First Affiliated Hospital of Guangzhou Medical College, Guangzhou 510120, China \\ ${ }^{3}$ Department of Oral \& Maxillofacial Surgery, The First Affiliated Hospital, College of Medicine, Zhejiang University, \\ Hangzhou 310003, China
}

Correspondence should be addressed to Zhiyong Li; hxlzy2002@163.com and Lei Liu; drliulei@163.com

Received 14 December 2014; Accepted 19 January 2015

Academic Editor: Wei Seong Toh

Copyright (C) 2015 Yuanwei Chen et al. This is an open access article distributed under the Creative Commons Attribution License, which permits unrestricted use, distribution, and reproduction in any medium, provided the original work is properly cited.

\begin{abstract}
Tooth regeneration is considered to be an optimistic approach to replace current treatments for tooth loss. It is important to determine the most suitable seed cells for tooth regeneration. Recently, human umbilical cord mesenchymal stem cells (hUCMSCs) have been regarded as a promising candidate for tissue regeneration. However, it has not been reported whether hUCMSCs can be employed in tooth regeneration. Here, we report that hUCMSCs can be induced into odontoblast-like cells in vitro and in vivo. Induced hUCMSCs expressed dentin-related proteins including dentin sialoprotein (DSP) and dentin matrix protein-1 (DMP-1), and their gene expression levels were similar to those in native pulp tissue cells. Moreover, DSP- and DMP-1-positive calcifications were observed after implantation of hUCMSCs in vivo. These findings reveal that hUCMSCs have an odontogenic differentiation potency to differentiate to odontoblast-like cells with characteristic deposition of dentin-like matrix in vivo. This study clearly demonstrates hUCMSCs as an alternative therapeutic cell source for tooth regeneration.
\end{abstract}

\section{Introduction}

Tooth loss caused by caries, periodontitis, and mechanical trauma is a major public health problem worldwide [1]. People with tooth loss have poor oral health-related quality of life involving the problems with eating, chewing, smiling, and communication [2]. Current treatments for tooth loss rely on artificial dentures, such as fixed bridges, removable dentures, and dental implants. However, compared with natural teeth, artificial dentures are nonbiological and have some disadvantages including a foreign body sensation and finite usability, all of which dissatisfy patients $[3,4]$. Thus, biological teeth are considered to be necessary [5].

In the fields of stem cells and tissue regeneration, biological tooth crowns and roots have already been generated in animal studies $[6,7]$. Moreover, tooth regeneration has been thought as a possible approach in the next generation of dental treatments [8]. However, one of key factors to achieve the goal is elucidation of the most suitable seed cells for tooth regeneration. Embryonic stem cells (ESCs) and adult stem cells are the two main types of stem cells for tooth regeneration [9]. Despite high proliferation and differentiation capabilities, ESCs are rarely applied in clinical practice because of possible tumorigenesis and ethical issues [10]. Thus, recent studies on seed cells for tooth regeneration have mainly focused on adult stem cells. Among the adult stem cells, dental stem cells have been considered as a candidate for tooth regeneration. These include the dental pulp stem cells, stem cells from exfoliated deciduous teeth, periodontal ligament stem cells, stem cells from apical papilla, and dental follicle progenitor cells [11]. All of these cells have already been proved owning multipotent and odontogenic differentiation potentials, and some of them have successfully applied into tooth regeneration studies [12]. However, the use of dental stem cells has several potential limitations. The primary challenge is the limited availability of dental stem 
cells, especially from those who are agomphious. In addition, cellular rejection and ethical issues in allogeneic therapy further hinder the clinical application of dental stem cells $[13,14]$. On the other hand, induction of nondental ectomesenchyme odontogenesis by coculture with oral epithelium has provided the experimental basis to use nondental adult stem cells for tooth regeneration [15]. Li et al. reported bone marrow mesenchymal stem cells (BMMSCs) produce tooth-like structures after coculture with oral epithelial cells derived from rat embryos [16], suggesting the possibility of tooth regeneration using BMMSCs. However, invasive and painful procedures to harvest BMMSCs are difficult for people including those who require dental treatment. Therefore, a more practical and suitable kind of seed cells is needed for tooth regeneration.

Since Romanov et al. isolated the mesenchymal stem cells (MSCs) from the human umbilical cord [17], these stem cells have gained significant attention, and many advantages of hUCMSCs have been recognized. Firstly, hUCMSCs are multipotent with high capabilities for differentiation and proliferation [18]. Secondly, there is no limitation of cell source for hUCMSCs, which is a major hurdle for other stem cell types. In fact, human cord blood banks have been established worldwide, which provide a reliable source of hUCMSCs [19, 20]. More importantly, human umbilical cords would otherwise be discarded after childbirth, and there are no invasive and/or painful procedures for both the mother and infant during collection, so there are fewer ethical issues [21]. In addition, because of the protection of the placental barrier, there is a lower risk of viral contamination compared with other sources of adult stem cells [22].

hUCMSCs can differentiate into cardiomyocytes, skeletal muscle cells, endotheliocytes, and neurons and have been applied in studies of osteochondral, musculoskeletal, and bone tissue regeneration [23-26]. However, it has not been reported whether hUCMSCs can be applied in tooth regeneration.

Therefore, in this study, we examined whether hUCMSCs have an odontogenic differentiation potential. We used Sprague-Dawley (SD) rat tooth germ cell conditioned medium (TGC-CM) and human tooth dentin matrix (hTDM) to induce the hUCMSCs into odontoblast-like cells in vitro and determined whether the hUCMSCs can be differentiated into odontoblast-like cells in vivo.

\section{Materials and Methods}

This study followed the guidelines in the Declaration of Helsinki and the International Guiding Principles for Animal Research and Law for Management of Experimental Animal. The Research Ethics Board for both human samples and animal experiments established by the Ethics Committee of the West China Hospital of Stomatology, Sichuan University, examined the proposed research protocol for this project and found it to be ethically acceptable.

2.1. hUCMSCs Isolation, Culture, and Identification. Fresh human umbilical cords were collected from full-term births by cesarean section from Chengdu Women's and Children's
Central Hospital and stored in phosphate buffered saline (PBS) containing $100 \mathrm{U} / \mathrm{mL}$ penicillin and $100 \mathrm{U} / \mathrm{mL}$ streptomycin, which was informed consent of the parents and conducted following approval of Sichuan University Ethics Committee. The human umbilical cords were processed within 4 hours and were assigned to testing groups and control groups at random. We applied collagenase/trypsin method $(0.2 \%$ collagenase and $0.25 \%$ trypsin, both purchased from Sigma, USA) and explant culture to isolated hUCMSCs from Wharton's jelly [27]. The cells were cultured by LGDMEM/F12 (Invitrogen, USA) containing 10\% fetal bovine serum (FBS, Gibco, USA), $100 \mathrm{U} / \mathrm{mL}$ penicillin (Hyclone, USA), and $100 \mathrm{U} / \mathrm{mL}$ streptomycin (Hyclone, USA), and the medium was changed every 2-3 days. Following 2-3 passages, immunohistochemistry was performed as per published protocols [28]. For adipogenic differentiation, passage three (P3) hUCMSCs were seeded at a density of $1 \times 10^{4}$ cells/well in 6-well plates and washed with PBS twice when the cells reached $80 \%$ confluence. The cells were maintained in LG-DMEM/F12 containing 10\% FBS, $1 \mu \mathrm{M}$ dexamethasone (Sigma, USA), $5 \mu \mathrm{g}$ insulin (Sigma, USA), $0.5 \mathrm{mM} 3$-isobutyl1-methylxanthine (Sigma, USA), and $0.2 \mathrm{mM}$ indomethacin (Sigma, USA). Half medium change was performed every 23 days. The cells were induced for 2 weeks, stained with Oil red O (Sigma, USA), and then observed under a microscope (CKX41, Olympus, Japan). For osteogenic differentiation, P3 hUCMSCs were seeded at a density of $1 \times 10^{4}$ cells/well in 6-well plates and cultured in LG-DMEM/F12 containing $10 \%$ FBS for 2 days and washed with PBS twice. Then, the cells were maintained in the osteogenic induction medium consisting of LG-DMEM/F12 containing 10\% FBS, $10 \mathrm{mM} \beta$ glycerophosphate (Sigma, USA), $10^{-8} \mathrm{~mol} / \mathrm{L}$ dexamethasone, and $50 \mu \mathrm{g} / \mathrm{mL}$ ascorbic acid (Sigma, USA). The medium was changed every 2-3 days for 3 weeks until a black opaque area was observed under a microscope and white nodules were observed by the naked eye. Alizarin red S staining was applied to detect the calcium nodules.

2.2. Differentiation of hUCMSCs into Odontoblast-Like Cells with TGC-CM In Vitro. The isolation of tooth germs of $\mathrm{SD}$ rats and preparation of TGC-CM were performed as described previously $[28,29]$. Briefly, The mandibular first molar germs were dissected from postnatal 0.5 neonatal SD rats, diced into cubes of about $1 \mathrm{~mm}^{3}$, digested with $0.2 \%$ collagenase for $40-60 \mathrm{~min}$ at $37^{\circ} \mathrm{C}$, and neutralized with $\alpha$-MEM (Hyclone, USA) containing $10 \% \mathrm{FBS}, 100 \mathrm{U} / \mathrm{mL}$ penicillin, and $100 \mathrm{U} / \mathrm{mL}$ streptomycin. The digested cells were cultured by culture medium $\alpha$-MEM containing 10\% FBS, $100 \mathrm{U} / \mathrm{mL}$ penicillin, and $100 \mathrm{U} / \mathrm{mL}$ streptomycin. The medium was changed 2-3 days. The substituted conditioned medium was collected and centrifuged at $1000 \mathrm{rpm}$ for 5 minutes. The supernatant was passed through a $0.22 \mu \mathrm{m}$ bacterial filter and mixed with LG-DMEM/F12 (Invitrogen, USA) containing $10 \% \mathrm{FBS}, 100 \mathrm{U} / \mathrm{mL}$ penicillin, and $100 \mathrm{U} / \mathrm{mL}$ streptomycin at $1: 1$ ratio to obtain the TGC-CM.

Passage 2 (P2) hUCMSCs were seeded at a density of $1 \times$ $10^{4}$ cells/well in 6-well dishes and cultured for 24 hours. 
After washing the cells with PBS three times, the TGCCM was added to induce the hUCMSCs into odontoblastlike cells. The TGC-CM was changed every other day and the morphological changes of the cells were photographed under a microscope. Control hUCMSCs were cultured in LGDMEM/F12 containing 10\% FBS, $100 \mathrm{U} / \mathrm{mL}$ penicillin, and $100 \mathrm{U} / \mathrm{mL}$ streptomycin.

\subsection{Preparation of Human Tooth Dentin Matrix (hTDM).} TDM was obtained from healthy single root premolars extracted for orthodontic reasons with informed consent of the patients. And the procedures to treat the teeth were processed by previous study [30]. Briefly, the roots of donated teeth were cut half and stored in sterile deionized water for 56 hours and then were oscillated by ultrasonicator at $80 \mathrm{~Hz}$ for 5-6 minutes before changing the sterile deionized water every hour. Then the roots were treated in 17\%, 10\% and 5\% EDTA each for 6 minutes to remove the smear layer and rinsed with sterile deionized water for $5 \mathrm{~min}$ and then immersed in PBS containing $100 \mathrm{U} / \mathrm{mL}$ penicillin and $100 \mathrm{U} / \mathrm{mL}$ streptomycin for 72 hours following rinsing with sterile deionized water for 5 min and stored in LG-DMEM/F12 culture medium containing $100 \mathrm{U} / \mathrm{mL}$ penicillin and $100 \mathrm{U} / \mathrm{mL}$ streptomycin. After this, hematoxylin and eosin (HE) staining was performed to check whether the fiber tracts in hTDM became loose and the smear layer had been removed. And Masson's trichrome staining was performed to detect whether collagen fibers still existed in hTDM. And for MTT assay, the P2 hUCMSCs were seeded at a density of $5 \times 10^{3}$ cells/well in 24 -well dishes which contained hTDM, and the cells were cultured for 1-8 days. MTT solution $(40 \mu \mathrm{L})$ was added to each well, and the cells were incubated at $37^{\circ} \mathrm{C}$ in a humidified atmosphere with $5 \%$ $\mathrm{CO}_{2}$ for $3.5 \mathrm{~h}$. Then, the medium was aspirated, and $200 \mu \mathrm{L}$ of dimethyl sulfoxide was added to dissolve the blue crystals that formed in the cells. After gentle agitation for $10 \mathrm{~min}, 100 \mu \mathrm{L}$ of the solution in each well was transferred to a 96-well plate. The optical density was determined with a multiplate reader at a wavelength of $570 \mathrm{~nm}$. Control hUCMSCs were cultured without hTDM.

\subsection{Differentiation of hUCMSCs under the Odontogenic} Microenvironment Provided by the hTDM In Vitro and Vivo. For in vitro study, hUCMSCs were seeded at a density of $5 \times 10^{4}$ cells/well in 6-well plates containing hTDM, and negative control cells were seeded at a same density in 6-well plates without hTDM. Both of testing and control groups were cultured in culture medium LG-DMEM/F12 containing 10\% FBS, $100 \mathrm{U} / \mathrm{mL}$ penicillin, and $100 \mathrm{U} / \mathrm{mL}$ streptomycin. The culture medium was changed each other day and the cells were harvested after 14 days for analyses. For in vivo study, the hUCMSCs were seeded at a density of $5 \times 10^{4}$ cells/well in 6well plates containing hTDM and cultured in LG-DMEM/F12 for 24 hours at first. Then twenty hTDM-hUCMSC composites were implanted subcutaneously into the backs of nude mice under anesthesia. After 8 weeks, the implants were extracted and subjected to visual observation. HE staining, Masson's trichrome staining, and immunohistochemistry were performed to evaluate odontogenic differentiation of hUCMSCs in vivo.

2.5. Immunocytochemistry. For immunocytochemical analysis, the cells were fixed with $4 \%$ paraformaldehyde for 15 minutes. Immunocytochemistry was performed with streptavidin-biotin complex method according to the manufacturer's protocol. Antibodies against CD105 (1:100), CD29 (1:100), CD44 (1:100), CD34 (1:100), CD45 (1:100), CD31 $(1: 100)$, DSP $(1: 200)$, and DMP1 $(1: 100)$ were used in this study. The antibodies against DSP and DMP1 were purchased from Santa Cruz (USA). Other antibodies were purchased from ZSGB-BIO (China). Samples were photographed under an Olympus CKX41 microscope.

2.6. Western Blotting. hUCMSCs were collected and washed with PBS. Then, the cells were lysed with lysis buffer for 30 minutes. The proteins were separated by $10 \%$ SDSpolyacrylamide gel electrophoresis and then transferred to cellulose membranes. The membranes were incubated with gentle agitation at $37^{\circ} \mathrm{C}$ with primary antibodies $\beta$-actin ( $1: 1000$, Santa Cruz, USA), DMP-1 (1:100, Santa Cruz, USA), and DSP (1:200, Santa Cruz, USA). Then the membranes were incubated with gentle agitation for 2 hours at $37^{\circ} \mathrm{C}$ with horseradish peroxidase-conjugated secondary antibody diluted in 5\% skim milk powder at 1:7500. After washing in Tris-Buffered Saline Tween-20 (Beyotime, China) for three times (10 minutes each wash), the membranes were developed by an ECL western blotting detection system. Immunoreactive proteins were then detected by ChemiDoc MP System \#170-8280 (Bio-Rad, USA). Images were captured and analyzed by Quantity One software (Bio-Rad, USA).

2.7. Quantitative PCR. Total RNA was extracted with RNAiso Reagent (TaKaRa, Japan). The RNA was reversed-transcribed to cDNA using PrimeScript RT reagent Kit Perfect Real Time (TaKaRa, Japan). Quantitative PCR was performed in ABI PRISM 7300 Sequence Detection System (Applied Biosystems, USA). The relative expression levels for the target gene were evaluated using the $2^{-\Delta \Delta \mathrm{CT}}$ method [31]. $\beta$-actin gene expression was used for normalization of each sample. The primer pairs used for RT-qPCR were showed in Table 1.

2.8. Scanning Electron Microscope (SEM). hTDM was observed by scanning electron microscope (SEM) (Inspect F, FEI, Netherlands). Briefly, hTDM was washed with PBS for three times, and then it was fixed with $2.5 \%$ glutaraldehyde at $0^{\circ} \mathrm{C}$ and dehydrated and dried in a critical-point dryer. Finally, it was observed and photographed by SEM.

2.9. Statistical Analysis. All quantitative data are expressed as the mean \pm SD. Statistical analyses were performed using oneway analysis of variance using SPSS software. A value of $P<$ 0.05 was considered to be statistically significant.

\section{Results}

3.1. Isolation, Culture, and Identification of hUCMSCs. hUCMSCs isolated from Wharton's jelly were maintained 
TABLE 1: Forward (F) and reverse (R) primer sequences for target and reference genes.

\begin{tabular}{|c|c|c|c|}
\hline Gene name & Primer sequence $\left(5^{\prime}-3^{\prime}\right)$ & Fragment length (bp) & Accession number \\
\hline \multirow{2}{*}{$\beta$-actin } & F: GAAGATCAAGATCATTGCTCCT & \multirow[t]{2}{*}{111} & \multirow[t]{2}{*}{ NM_031144.2 } \\
\hline & R: TACTCCTGCTTGCTGATCCA & & \\
\hline \multirow{2}{*}{ DMP-1 } & F: AAGATCAGCATCCTGCTCAT & \multirow{2}{*}{91} & \multirow{2}{*}{ NM_004407.3 } \\
\hline & R: CTTCAGAATCCTCAGATTCAT & & \\
\hline \multirow{2}{*}{ DSPP } & F: GAATAGAGGACACCCAGAAG & \multirow{2}{*}{165} & \multirow{2}{*}{ NM_014208.3 } \\
\hline & R: CTTTCCCAACTTCTTTGGTAAT & & \\
\hline
\end{tabular}

under standard culture conditions, and the primary and passaged cells both exhibited adherence to plastic (Figure 1(a)). Immunohistochemistry showed that the adherent cells were stained positively for mesenchymal markers CD29, CD44, and CD105 but were negative for hematopoietic lineage markers CD34 and CD45 and endothelial cell markers CD31 (Figure 1(b)). After adipogenic induction for 2 weeks, lipid droplets were found in the cytoplasm, indicating that the cells had differentiated into the fat cells (Figure 1(c)). In addition, during the osteogenic induction, there was little change in cell morphology, but refractile substances were observed in the cell colonies. Calcium accumulation was found after being induced for 2 weeks as small round Alizarin red-positive nodules in the cells (Figure $1(\mathrm{~d})$ ). Thus, the hUCMSCs demonstrated multipotency.

3.2. hUCMSCs Have an Odontogenic Differentiation Potential. After the hUCMSCs had been cultured in TGC-CM for 14 days, the cells grew well and exhibited a long fusiform shape with abundant cytoplasm, but the morphology did not change significantly during the induction procedure (Figure 2(a)). We also found that TGC-CM-induced hUCMSCs expressed both DSP and DMP-1 detected by immunocytochemistry and western blotting. These proteins were not expressed in uninduced hUCMSCs but were found in pulp tissue, indicating that the TGC-CM-induced hUCMSCs differentiated into odontoblast-like cells (Figures 2(b) and 2(c)). Next, quantitative PCR was used to compare the gene expression of DSSP and DMP-1 in TGC-CM induced hUCMSCs and uninduced hUCMSCs. We found that DSSP and $D M P-1$ gene expression was upregulated significantly in both TGC-CM-induced hUCMSCs and pulp tissue, whereas no expression was found in uninduced hUCMSCs (Figures 2(d) and 2(e)).

3.3. hTDM Provides an Odontogenic Microenvironment for hUCMSCs. The sectioned hTDM was stained with HE and Masson's trichrome. HE staining revealed loose fiber tracts on the surface of the prepared hTDM (Figure 3(a)). Masson's trichrome staining of the prepared hTDM was dark red with gradual darker blue from distal to proximal pulp cavity dentin, where collagen fibers existed from low to high abundance (Figure 3(b)). SEM observation further confirmed that the dentin tubules were fully exposed and the loose peritubular and intertubular fibers provided the space where hUCMSCs could keep contact with proteins and factors involved in dentin formation and thus provided an odontogenic microenvironment for the hUCMSCs (Figure 3(c)).
Next, immunohistochemistry was used to determine whether DSP and DMP-1 were expressed in hTDM. As expected, hTDM was positive for DSP and DMP-1, especially around the dentin tubules, indicating that the dentin expressed DSP and DMP-1 (Figure 3(d)).

The growth curve detected by MTT assays of hTDMinduced hUCMSCs and normal cultured hUCMSCs without hTDM were similar. Both showed a latent phase for 24 to 36 hours, a logarithmic phase for 5 or 6 days, and a plateau phase after 7 days of culture (Figure 3(e)). And the SEM showed the hUCMSCs had adhered to the hTDM surface after induction for 2 hours, and then the cells began to spread after 24 hours and the whole hTDM surface was covered by cells after 7 days. These observations demonstrated that the hTDM had no effect on proliferation of the hUCMSCs (Figure 3(f)).

Then immunocytochemistry and western blotting proved DSP and DMP-1 exist in hTDM-induced hUCMSCs (Figures 4(a) and 4(b)). We also found that hTDM-induced hUCMSCs expressed DSPP and DMP-1, whereas the uninduced hUCMSCs did not express these genes. Despite the low level of DSPP expression, the differences in DSPP and DMP-1 expression between hTDM-induced hUCMSCs and uninduced hUCMSCs were statistically significant (Figures 4(c) and 4(d)).

3.4. Dentin Regeneration by Subcutaneously Implanting hTDMhUCMSC Composites. To investigate whether hUCMSCs are suitable seed cells for tooth regeneration, we implanted hTDM-hUCMSC composites in vivo. The hTDM-hUCMSC composites were harvested after subcutaneous implantation into 20 nude mice for 8 weeks. We found no swelling or inflammation in the tissues around the implants. Furthermore, the implants maintained their original appearance without any degradation. HE staining showed newly formed calcification on the hTDM with multiple layers of cells but no inflammatory cells (Figure 5(a)). Masson's trichrome staining also showed newly formed calcification (Figure 5(b)). Importantly, the newly formed calcification and adherent cells were positive for DSP and DMP-1 as detected by immunohistochemistry (Figure 5(c)). These results showed that hUCMSCs can be induced into odontoblast-like cells by hTDM in vivo.

\section{Discussion}

The hUCMSCs can be isolated from various regions of the umbilical cord. These regions include Wharton's jelly, umbilical vein subendothelium, and the perivascular region [32]. Wharton's jelly is a primitive mucous connective tissue 


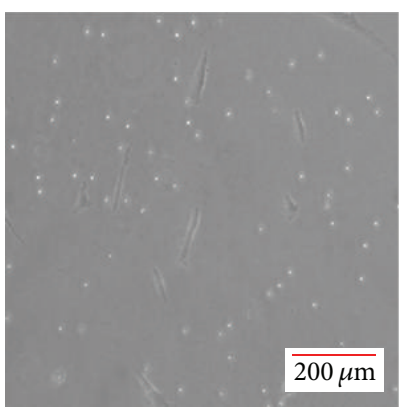

48 hours

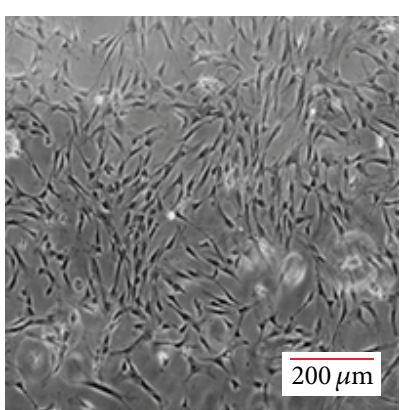

7 days

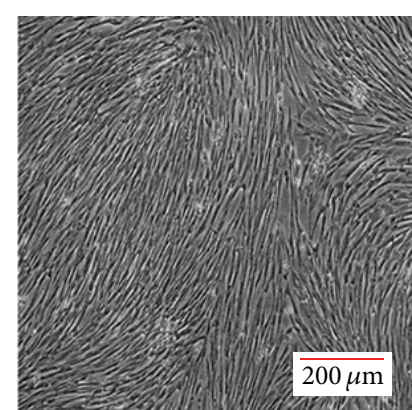

10 days

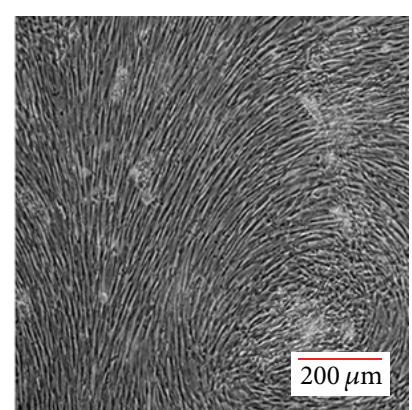

14 days

(a)

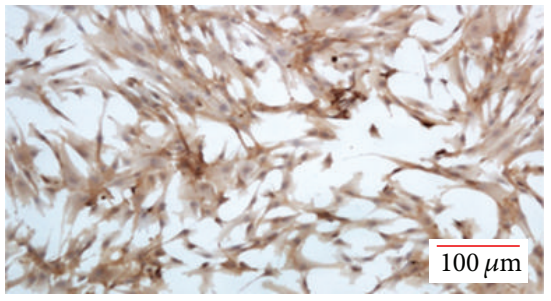

CD29(+)

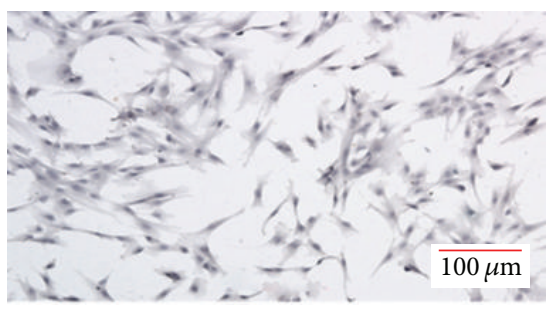

CD31(-)

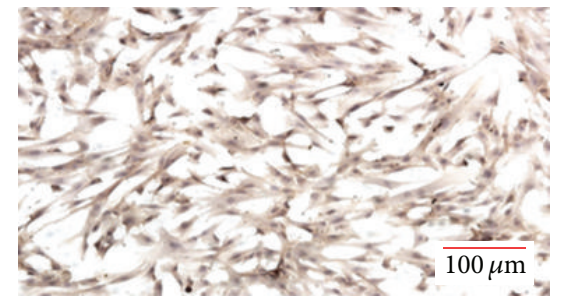

CD44(+)

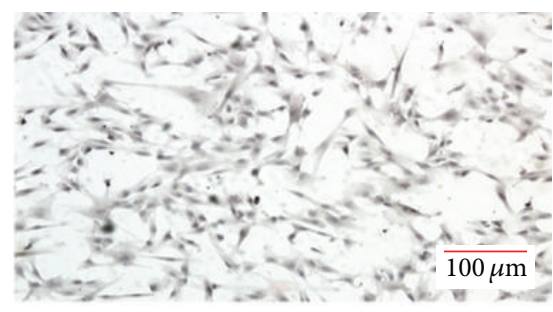

CD34(-)

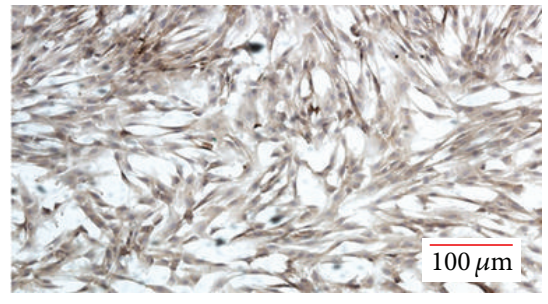

CD105(+)

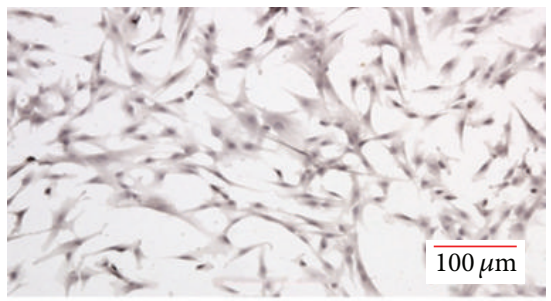

CD45(-)

(b)

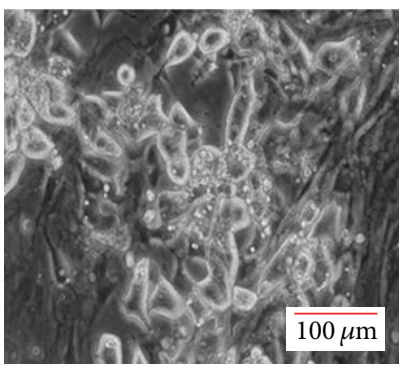

(c)

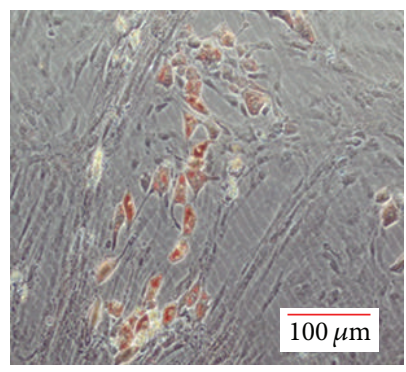

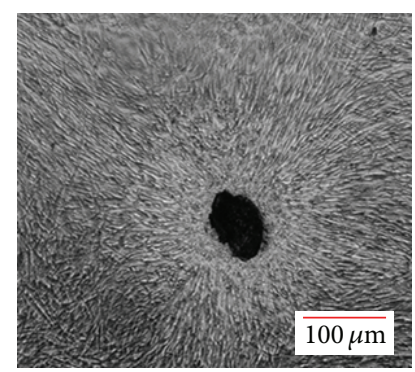

(d)

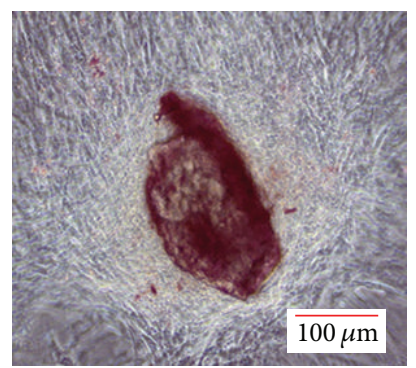

FIGURE 1: Isolation, culture, and identification of hUCMSCs. (a) Primary mesenchymal stem cells isolated from human Wharton's jelly formed colonies after 7 days, reached confluency after 10 days, and were in a vortex-like arrangement after 14 days. (b) The adherent cells were stained positively for mesenchymal markers CD29, CD44, and CD105 but were negative for hematopoietic lineage markers CD34 and CD45 and endothelial cell marker CD31. (c) After adipogenic induction for 2 weeks, vacuole-like changes were observed under a microscope (left, $\times 40$ ), and the cells were stained by oil red $\mathrm{O}$ (right). (d) After osteogenic induction, refractile substances were observed in cell colonies (left, $\times 40$ ), and small round nodules were detected by Alizarin red S staining (right).

that surrounds the umbilical cord arteries and vein. The mesenchymal stem cells from Wharton's jelly have been confirmed as a primitive stem cell population [33]. In addition, stem cells from Wharton's jelly have a better multiple differentiation potential than those from other regions of umbilical cord [34, 35]. Therefore, we isolated MSCs from Wharton's jelly of the human umbilical cord and applied them for tooth regeneration.
The niche plays an important role in stem cell proliferation and differentiation. Specific niches participate in regulating the asymmetric divisions of stem cells $[36,37]$. Thus, to induce hUCMSCs into odontoblast-like cells in vitro, it is important to provide a microenvironment that mimics the specific niche of tooth morphogenesis and facilitates odontogenic differentiation. Previous studies have demonstrated that TGC-CM provides a microenvironment enriched with 


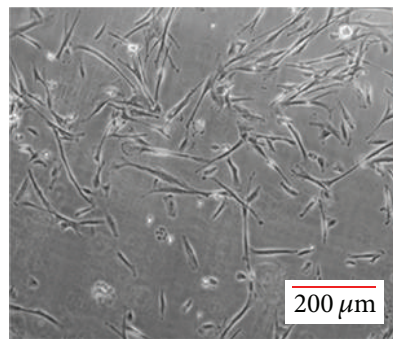

Preinduced

$\operatorname{DSP}(+)$

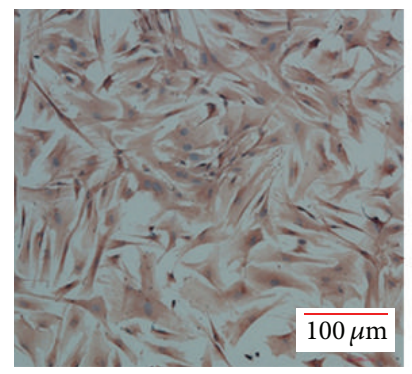

$\operatorname{DSP}(-)$

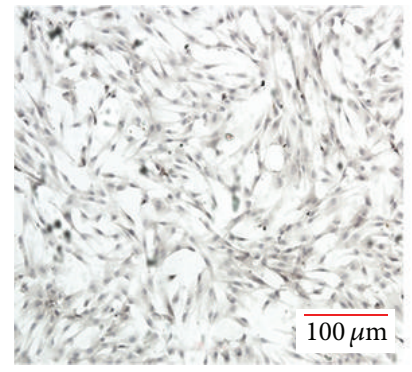

(b)

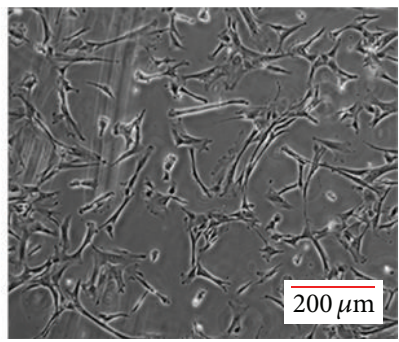

Induced for 3 days

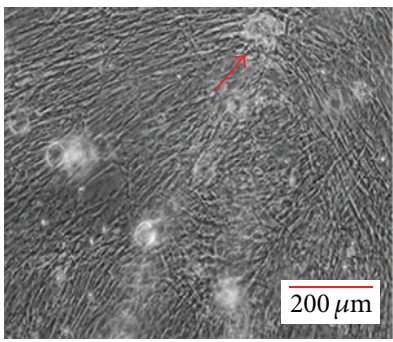

Induced for 14 days

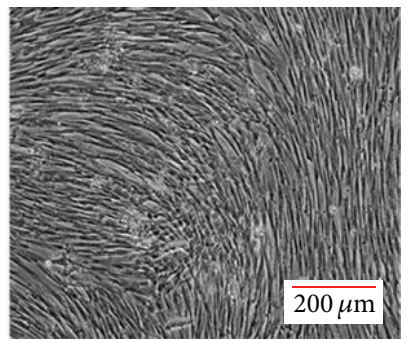

Uninduced P2 hUCMSCs

(a)

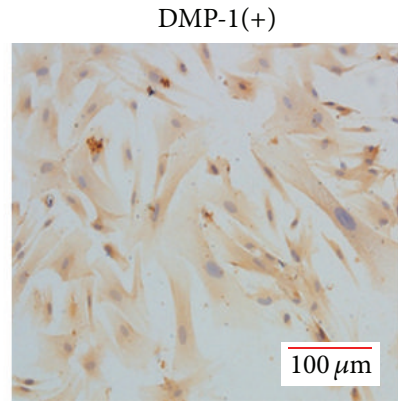

DMP-1(-)
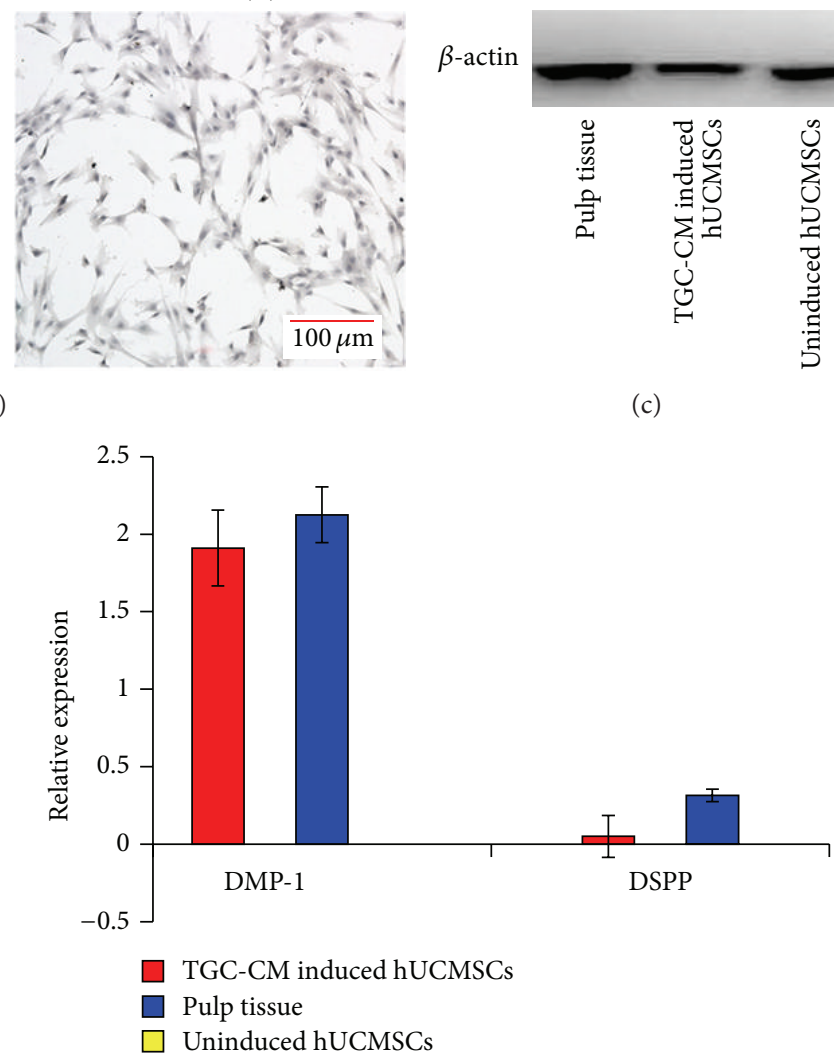

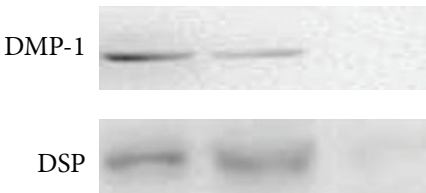

DMP-1
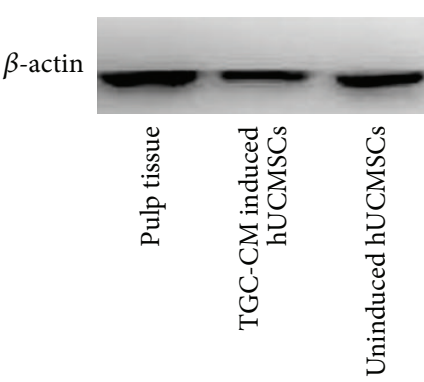

(c)

(d)

(e)

FIgURE 2: TGC-CM induces hUCMSCs into odontoblast-like cells in vitro. (a) After hUCMSCs were induced in TGC-CM for 3 days, there was little change in the cell morphology. After 14 days, the TGC-CM-induced hUCMSCs grew well and calcified areas were observed (arrow), but the morphology was similar to uninduced cells. (b) Odontoblast marker proteins DSP and DMP-1 were detected in TGC-CM-induced hUCMSCs by immunocytochemistry, but not in uninduced hUCMSCs. (c) Western blotting showed that TGC-CM-induced hUCMSCs and pulp tissue expressed DMP-1 and DSP, whereas uninduced hUCMSCs did not express these proteins. (d) and (e) Relative mRNA levels of DMP-1 and DSPP were determined by quantitative PCR. 

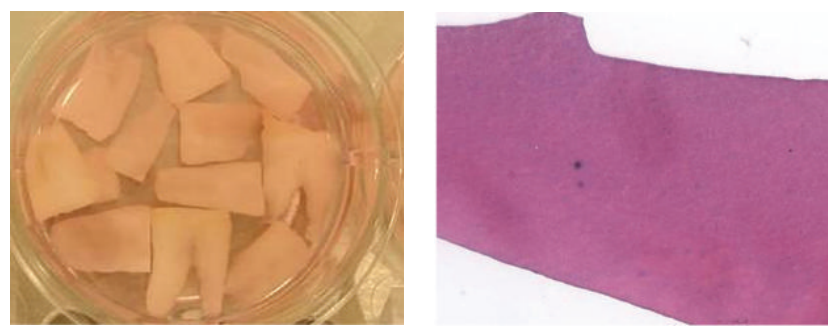

$\times 40$

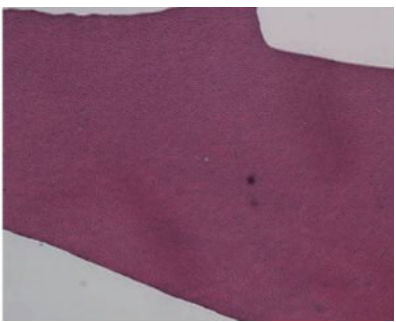

$\times 100$

(a)

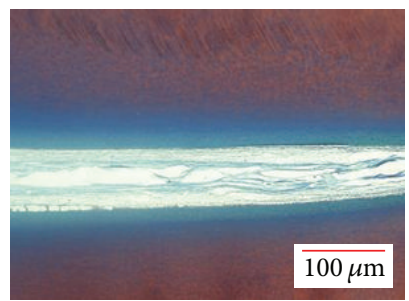

Normal tooth root

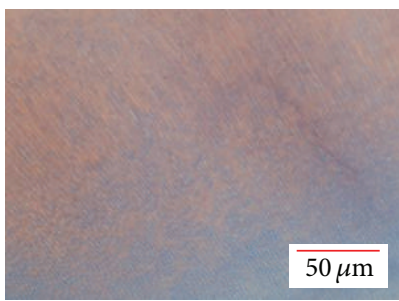

hTDM

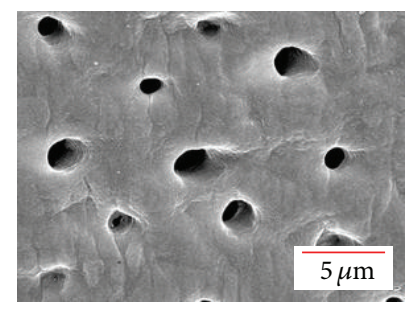

(b)
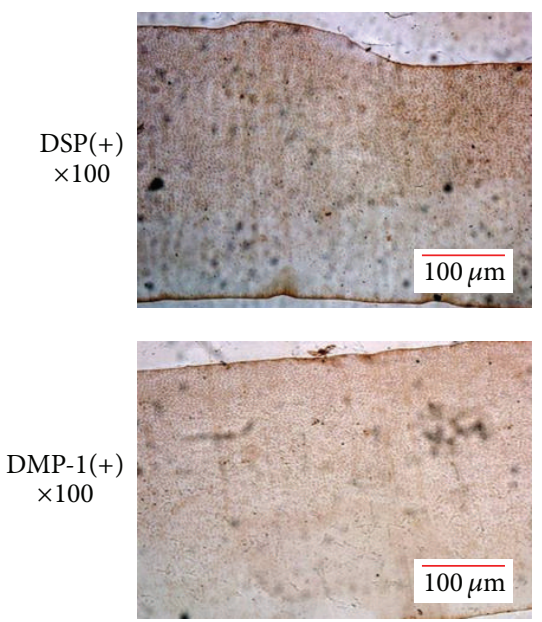

(d)

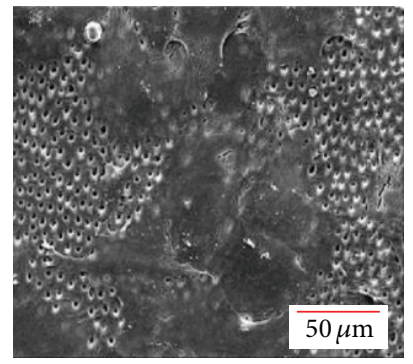

2 hours

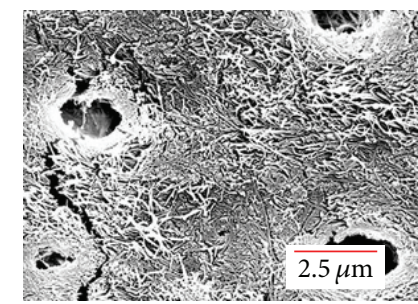

(c)

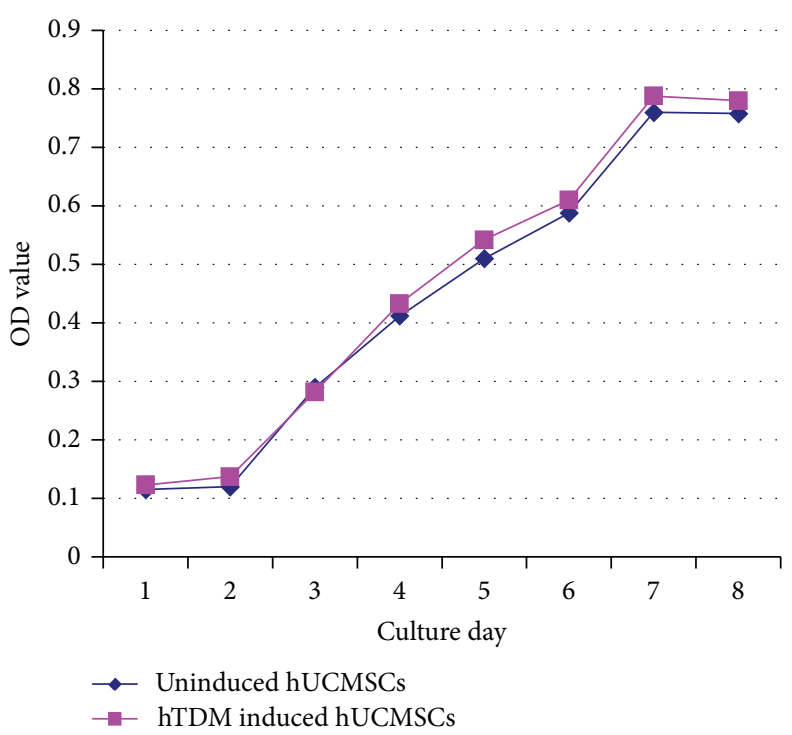

(e)

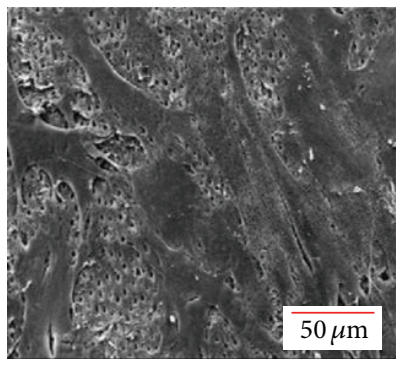

24 hours

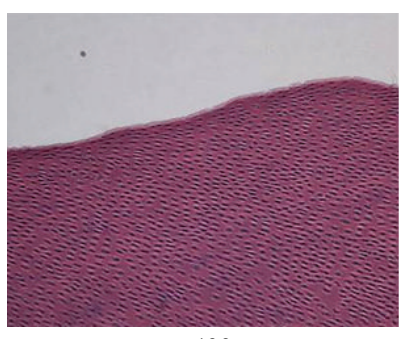

$\times 400$ 


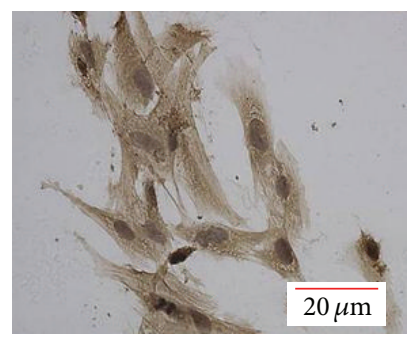

$\mathrm{DSP}(+) \times 400$

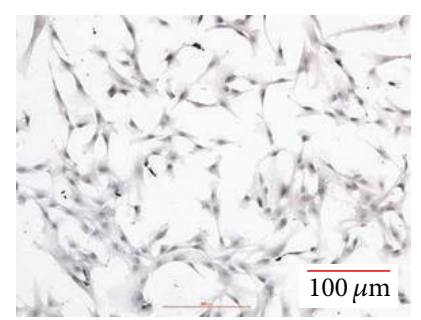

$\operatorname{DSP}(-) \times 100$

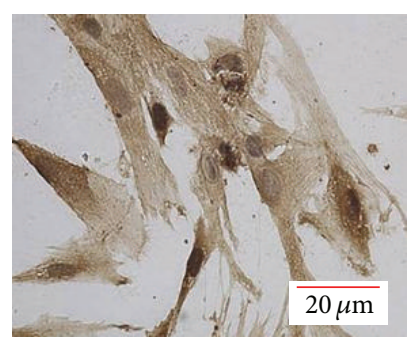

DMP-1(+) $\times 400$

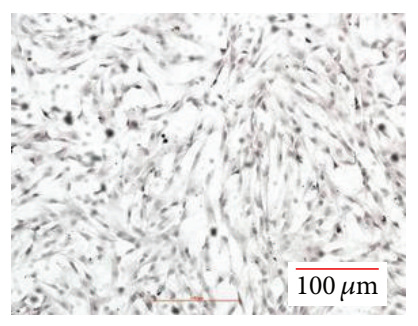

DMP-1(-) $\times 100$

(a)

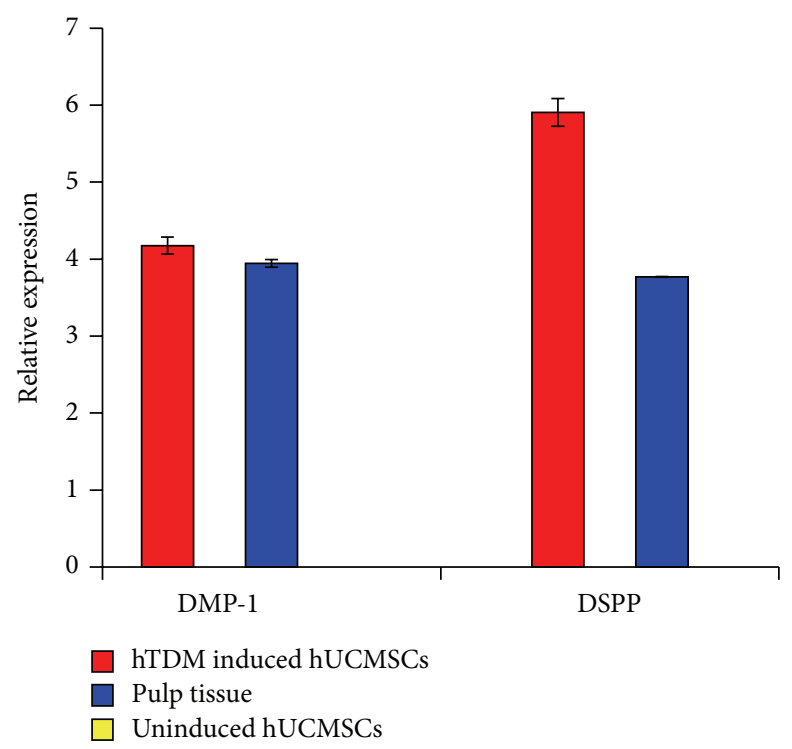

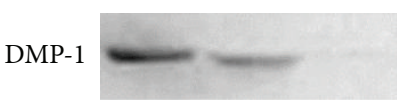

DMP-1

DSP

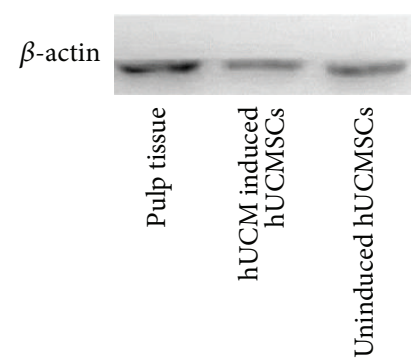

(b)

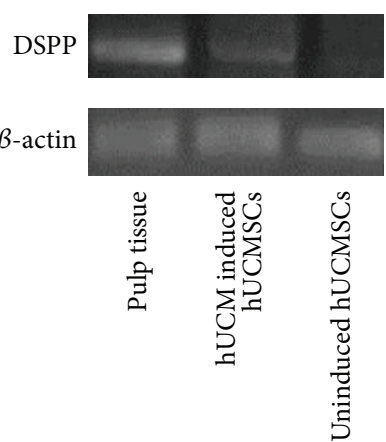

(c)

(d)

FIGURE 4: hTDM induces hUCMSCs into odontoblast-like cells in vitro. (a) Detection of DSP and DMP-1 in hTDM-induced hUCMSCs and normal cultured hUCMSCs without hTDM by immunocytochemistry. (b) Western blotting showed that hTDM-induced hUCMSCs and pulp tissue expressed DSP and DMP-1, while uninduced hUCMSCs did not express these proteins. (c) and (d) Relative mRNA levels of DMP-1 and DSPP were determined by quantitative PCR.

regulating factors for tooth morphogenesis, which enhanced odontogenic differentiation of dental as well as nondental stem cells $[38,39]$. Tooth morphogenesis is regulated by sequential and reciprocal interactions between the epithelial and mesenchymal tissues. Moreover, the signals from the oral epithelium play a decisive role in tooth morphogenesis, which act on ectomesenchyme cells to initiate and maintain tooth morphogenesis [40]. When developing tooth germ cells are cultured in the culture medium, the interactions between the epithelial and mesenchymal cells result in secretion of various factors including Wnt, fibroblast growth factor, transforming growth factor- $\beta$, and bone morphogenetic proteins [41, 42].
After inducing hUCMSCs with TGC-CM, these factors initiate and maintain odontogenic differentiation.

Tooth regeneration mainly focuses on three aspects: seed cells, scaffolds, and growth factors [43]. Seed cells for tooth regeneration should be able to differentiate into tooth-specific cells and form dentin, enamel, cementum, and alveolar bone in a suitable scaffold [44]. To determine whether hUCMSCs are suitable for tooth regeneration, we carried out in vitro and in vivo experiments using cocultured hUCMSCs with scaffolds. There are various scaffolds for tooth regeneration such as collagen, polyglycolic acid, and polylactic acid [45]. Among them, TDM is a newly developed scaffold for tooth 


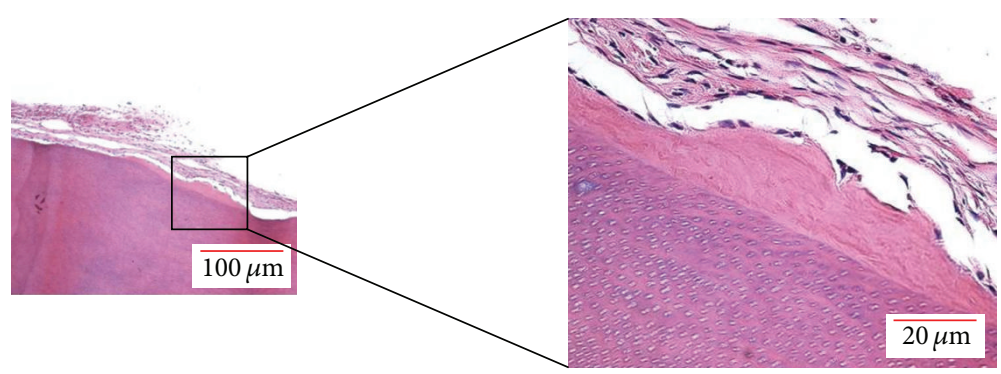

(a)

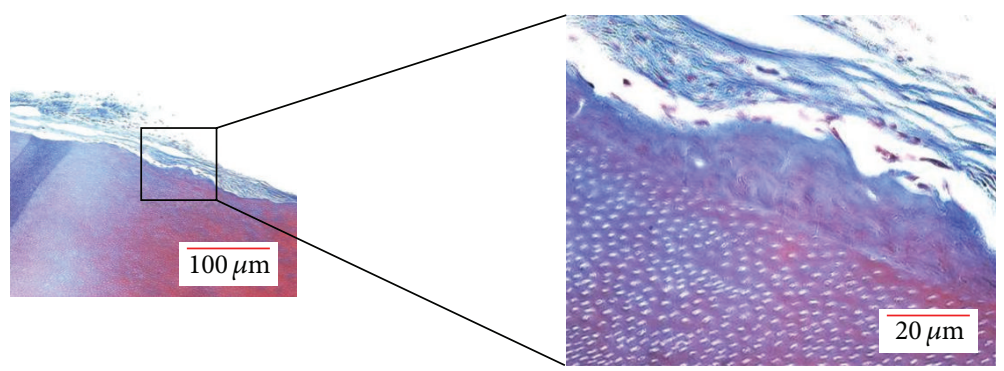

(b)
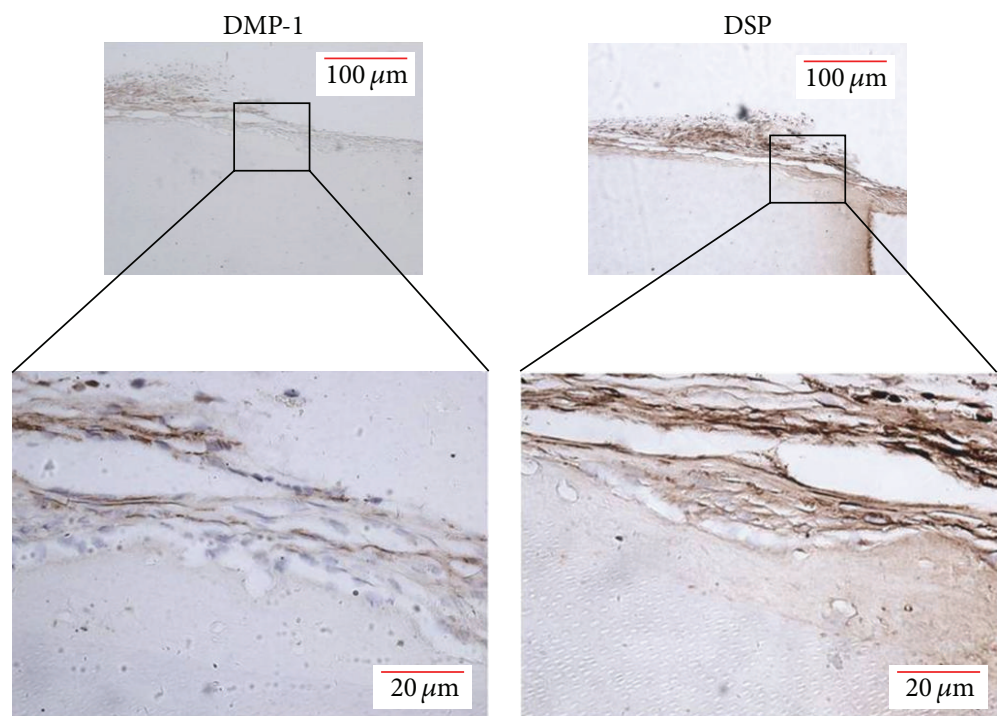

(c)

FIGURE 5: hTDM induces hUCMSCs into odontoblast-like cells in vivo. (a) HE staining of hTDM-hUCMSC composites after subcutaneous implantation into nude mice for 8 weeks. (b) Masson's tricolor staining of hTDM-hUCMSC composites after subcutaneous implantation into nude mice for 8 weeks. (c) Newly formed calcification and adhesive cells on hTDM were positive for DSP and DMP-1 as detected by immunohistochemistry.

regeneration. Prepared hTDM maintains the major structure of dentin tubules, which is essential for dentin regeneration. In addition, hTDM expressed DSP and DMP-1 which have been demonstrated to play critical roles in dentinogenesis [46]. Thus, hTDM not only serves as a scaffold, but also provides an odontoblastic microenvironment for stem cells $[30,47]$. Therefore, hTDM is regarded as an available scaffold for tooth regeneration. Our results showed that hUCMSCs can be differentiated into odontoblast-like cells by hTDM in vitro, and that the proliferation rate of hUCMSCs was not altered after combining with hTDM. Furthermore, newly formed calcifications were observed after hTDM-hUCMSC composites were implanted subcutaneously into nude mice for 8 weeks. Moreover, the newly formed calcifications were positive for DSP and DMP-1 as detected by immunohistochemistry, revealing that the newly formed calcifications were likely to be dentin-like matrix. And unsurprisingly, the hUCMSCs without hTDM in control groups could not differentiation into odontoblast-like cells whether in vitro or in vivo. Therefore, we concluded that hUCMSC can be induced into odontoblast-like cells that secrete a dentinlike matrix in vivo, suggesting that hUCMSCs are a suitable and practical cell type which could be applied in tooth regeneration. 
DSP (encoded by gene DSPP) is related to differentiation and mineralization of odontoblasts [48]. In addition, DMP-1 (encoded by the gene DMP-1) controls nucleation of calcium phosphate polymorphs and promotes pulp stem cell differentiation into odontoblasts [49]. DSP and DMP1 are generally regarded as odontoblast-specific markers to identify induction of odontoblast-like cells [50,51]. In the present study, hUCMSCs induced by TGC-CM or hTDM in vitro or in vivo were found to express DSP and DMP-1 with upregulation of DSPP and DMP-1 gene expression to levels similar to those in pulp tissue.

Although we have confirmed the hUCMSCs have an odontogenic differentiation potential, there are some limitations and challenges should be concerned. Although the sources of hUCMCSs are rich, the differentiation efficiency should be improved. And how to maintain cell stability and consistency should also be considered. For tooth regeneration research, further studies like distinguishing odontogenic differentiation from osteogenic differentiation are needed. And regenerating the whole tooth with hUCMSCs including periodontal ligament, dental pulp enamel, cementum, and dentin should also be studied in the near future.

\section{Conclusion}

In this study, we show that the hUCMSCs have an odontogenic differentiation potential to differentiate into odontoblast-like cells in an odontogenic microenvironment provided by TGC-CM and hTDM in vitro. Furthermore, hUCMSCs deposited a dentin-like matrix when combined with hTDM in vivo. Overall, hUCMSCs may be a new therapeutic cell source for tooth regeneration.

\section{Conflict of Interests}

The authors declare that there is no conflict of interests regarding the publication of this paper.

\section{Authors' Contribution}

Yuanwei Chen and Yongchun Yu contributed equally to this work.

\section{Acknowledgments}

Financial support was provided by National Natural Foundation of China (81070802, 81271096), Fundamental Research Funds for the Central Universities of China (2011SCUD4B14), and Public Application Research Funds for Science Technology Department of Zhejiang Provincial (no. 2012C33010).

\section{References}

[1] P. E. Petersen, D. Bourgeois, H. Ogawa, S. Estupinan-Day, and C. Ndiaye, "The global burden of oral diseases and risks to oral health," Bulletin of the World Health Organization, vol. 83, no. 9, pp. 661-669, 2005.

[2] A. E. Gerritsen, P. F. Allen, D. J. Witter, E. M. Bronkhorst, and N. H. J. Creugers, "Tooth loss and oral health-related quality of life: a systematic review and meta-analysis," Health and Quality of Life Outcomes, vol. 8, article 126, p. 552, 2010.

[3] A. Ćelebić and D. K. Zlatarić, "Factors related to patients' general satisfaction with removable partial dentures: a stepwise multiple regression analysis," International Journal of Prosthodontics, vol. 21, no. 1, pp. 86-88, 2008.

[4] F. McCord and R. Smales, "Oral diagnosis and treatment planning: part 7. Treatment planning for missing teeth," British Dental Journal, vol. 213, no. 7, pp. 341-351, 2012.

[5] S. Yildirim, S. Y. Fu, K. Kim et al., "Tooth regeneration: a revolution in stomatology and evolution in regenerative medicine," International Journal of Oral Science, vol. 3, no. 3, pp. 107-116, 2011.

[6] W. Sonoyama, Y. Liu, D. Fang et al., "Mesenchymal stem cellmediated functional tooth regeneration in Swine," PLOS ONE, vol. 1, no. 1, article e79, 2006.

[7] C. S. Young, S. Terada, J. P. Vacanti, M. Honda, J. D. Bartlett, and P. C. Yelick, "Tissue engineering of complex tooth structures on biodegradable polymer scaffolds," Journal of Dental Research, vol. 81, no. 10, pp. 695-700, 2002.

[8] Y. Wang, B. Preston, and G. Guan, "Tooth bioengineering leads the next generation of dentistry," International Journal of Paediatric Dentistry, vol. 22, no. 6, pp. 406-418, 2012.

[9] J. J. Mao, P. G. Robey, and D. J. Prockop, "Stem cells in the face: tooth regeneration and beyond," Cell Stem Cell, vol. 11, no. 3, pp. 291-301, 2012.

[10] K. Otsu, M. Kumakami-Sakano, N. Fujiwara et al., "Stem cell sources for tooth regeneration: current status and future prospects," Frontiers in Physiology, vol. 5, article 36, 2014.

[11] G. T.-J. Huang, S. Gronthos, and S. Shi, "Mesenchymal stem cells derived from dental tissues vs. those from other sources: their biology and role in regenerative medicine," Journal of Dental Research, vol. 88, no. 9, pp. 792-806, 2009.

[12] M. M. Cordeiro, Z. Dong, T. Kaneko et al., "Dental pulp tissue engineering with stem cells from exfoliated deciduous teeth," Journal of Endodontics, vol. 34, no. 8, pp. 962-969, 2008.

[13] C. Morsczeck, G. Schmalz, T. E. Reichert, F. Völlner, K. Galler, and O. Driemel, "Somatic stem cells for regenerative dentistry," Clinical Oral Investigations, vol. 12, no. 2, pp. 113-118, 2008.

[14] S. M. Lee, Q. Zhang, and A. D. Le, "Dental stem cells: sources and potential applications," Current Oral Health Reports, vol. 1, no. 1, pp. 34-42, 2014.

[15] M. Mina and E. J. Kollar, "The induction of odontogenesis in non-dental mesenchyme combined with early murine mandibular arch epithelium," Archives of Oral Biology, vol. 32, no. 2, pp. 123-127, 1987.

[16] Z.-Y. Li, L. Chen, L. Liu, Y.-F. Lin, S.-W. Li, and W.-D. Tian, "Odontogenic potential of bone marrow mesenchymal stem cells," Journal of Oral and Maxillofacial Surgery, vol. 65, no. 3, pp. 494-500, 2007.

[17] Y. A. Romanov, V. A. Svintsitskaya, and V. N. Smirnov, "Searching for alternative sources of postnatal human mesenchymal stem cells: candidate MSC-like cells from umbilical cord," Stem Cells, vol. 21, no. 1, pp. 105-110, 2003.

[18] S. Karahuseyinoglu, O. Cinar, E. Kilic et al., "Biology of stem cells in human umbilical cord stroma: in situ and in vitro surveys," Stem Cells, vol. 25, no. 2, pp. 319-331, 2007.

[19] W. Gong, Z. Han, H. Zhao et al., "Banking human umbilical cord-derived mesenchymal stromal cells for clinical use," Cell Transplantation, vol. 21, no. 1, pp. 207-216, 2012. 
[20] M. G. Butler and J. E. Menitove, "Umbilical cord blood banking: an update," Journal of Assisted Reproduction and Genetics, vol. 28, no. 8, pp. 669-676, 2011.

[21] N. Forraz and C. P. Mcguckin, "The umbilical cord: a rich and ethical stem cell source to advance regenerative medicine," Cell Proliferation, vol. 44, no. 1, pp. 60-69, 2011.

[22] P. Duya, Y. Bian, X. Chu, and Y. Zhang, "Stem cells for reprogramming: could hUMSCs be a better choice?" Cytotechnology, vol. 65, no. 3, pp. 335-345, 2013.

[23] L. Wang, L. Zhao, and M. S. Detamore, "Human umbilical cord mesenchymal stromal cells in a sandwich approach for osteochondral tissue engineering," Journal of Tissue Engineering and Regenerative Medicine, vol. 5, no. 9, pp. 712-721, 2011.

[24] L. Wang, L. Ott, K. Seshareddy, M. L. Weiss, and M. S. Detamore, "Musculoskeletal tissue engineering with human umbilical cord mesenchymal stromal cells," Regenerative Medicine, vol. 6, no. 1, pp. 95-109, 2011.

[25] W. Thein-Han and H. H. K. Xu, "Collagen-calcium phosphate cement scaffolds seeded with umbilical cord stem cells for bone tissue engineering," Tissue Engineering Part A, vol. 17, no. 23-24, pp. 2943-2954, 2011.

[26] Y.-S. Fu, Y.-T. Shih, Y.-C. Cheng, and M.-Y. Min, “Transformation of human umbilical mesenchymal cells into neurons in vitro," Journal of Biomedical Science, vol. 11, no. 5, pp. 652-660, 2004.

[27] P. Salehinejad, N. B. Alitheen, A. M. Ali et al., "Comparison of different methods for the isolation of mesenchymal stem cells from human umbilical cord Wharton's jelly," In Vitro Cellular \& Developmental Biology. Animal, vol. 48, no. 2, pp. 75-83, 2012.

[28] J. Yu, Z. Deng, J. Shi et al., "Differentiation of dental pulp stem cells into regular-shaped dentin-pulp complex induced by tooth germ cell conditioned medium," Tissue Engineering, vol. 12, no. 11, pp. 3097-3105, 2006.

[29] M. T. Duailibi, S. E. Duailibi, C. S. Young, J. D. Bartlett, J. P. Vacanti, and P. C. Yelick, "Bioengineered teeth from cultured rat tooth bud cells," Journal of Dental Research, vol. 83, no. 7, pp. 523-528, 2004.

[30] R. Li, W. Guo, B. Yang et al., "Human treated dentin matrix as a natural scaffold for complete human dentin tissue regeneration," Biomaterials, vol. 32, no. 20, pp. 4525-4538, 2011.

[31] K. J. Livak and T. D. Schmittgen, "Analysis of relative gene expression data using real-time quantitative PCR and the $2^{-\Delta \Delta C_{\mathrm{T}}}$ method," Methods, vol. 25, no. 4, pp. 402-408, 2001.

[32] D. L. Troyer and M. L. Weiss, "Concise review: wharton's jellyderived cells are a primitive stromal cell population," Stem Cells, vol. 26, no. 3, pp. 591-599, 2008.

[33] H.-S. Wang, S.-C. Hung, S.-T. Peng et al., "Mesenchymal stem cells in the Wharton's jelly of the human umbilical cord," Stem Cells, vol. 22, no. 7, pp. 1330-1337, 2004.

[34] D. T. Harris, "Umbilical cord tissue mesenchymal stem cells: characterization and clinical applications," Current Stem Cell Research \& Therapy, vol. 8, no. 5, pp. 394-399, 2013.

[35] M. T. Conconi, R. di Liddo, M. Tommasini, C. Calore, and P. P. Parnigotto, "Phenotype and differentiation potential of stromal populations obtained from various zones of human umbilical cord: an overview," The Open Tissue Engineering and Regenerative Medicine Journal, vol. 4, no. 1, pp. 6-20, 2011.

[36] D. T. Scadden, "Nice neighborhood: emerging concepts of the stem cell niche," Cell, vol. 157, no. 1, pp. 41-50, 2014.

[37] L. Li and W. B. Neaves, "Normal stem cells and cancer stem cells: the niche matters," Cancer Research, vol. 66, no. 9, pp. 4553$4557,2006$.
[38] Y.-X. Wang, Z.-F. Ma, N. Huo et al., "Porcine tooth germ cell conditioned medium can induce odontogenic differentiation of human dental pulp stem cells," Journal of Tissue Engineering and Regenerative Medicine, vol. 5, no. 5, pp. 354-362, 2011.

[39] N. Huo, L. Tang, Z. Yang et al., "Differentiation of dermal multipotent cells into odontogenic lineage induced by embryonic and neonatal tooth germ cell-conditioned medium," Stem Cells and Development, vol. 19, no. 1, pp. 93-103, 2010.

[40] T. Nakamura, Y. Yamada, and S. Fukumoto, "Review: the regulation of tooth development and morphogenesis," in Interface Oral Health Science 2011, pp. 14-21, Springer, Tokyo, Japan, 2012.

[41] M. Jussila and I. Thesleff, "Signaling networks regulating tooth organogenesis and regeneration, and the specification of dental mesenchymal and epithelial cell lineages," Cold Spring Harbor Perspectives in Biology, vol. 4, no. 4, Article ID a008425, 2012.

[42] M. Tummers and I. Thesleff, "The importance of signal pathway modulation in all aspects of tooth development," Journal of Experimental Zoology Part B: Molecular and Developmental Evolution, vol. 312, no. 4, pp. 309-319, 2009.

[43] L. Peng, L. Ye, and X.-D. Zhou, "Mesenchymal stem cells and tooth engineering," International Journal of Oral Science, vol. 1, no. 1, pp. 6-12, 2009.

[44] G. Bluteau, H.-U. Luder, C. De Bari, and T. A. Mitsiadis, "Stem cells for tooth engineering," European Cells and Materials, vol. 16, pp. 1-9, 2008.

[45] L. Zhang, Y. Morsi, Y. Wang, Y. Li, and S. Ramakrishna, "Review scaffold design and stem cells for tooth regeneration," Japanese Dental Science Review, vol. 49, no. 1, pp. 14-26, 2013.

[46] A. Rangiani, Z.-G. Cao, Y. Liu et al., "Dentin matrix protein 1 and phosphate homeostasis are critical for postnatal pulp, dentin and enamel formation," International Journal of Oral Science, vol. 4, no. 4, pp. 189-195, 2013.

[47] W. Guo, Y. He, X. Zhang et al., "The use of dentin matrix scaffold and dental follicle cells for dentin regeneration," Biomaterials, vol. 30, no. 35, pp. 6708-6723, 2009.

[48] S. Suzuki, N. Haruyama, F. Nishimura, and A. B. Kulkarni, "Dentin sialophosphoprotein and dentin matrix protein-1: two highly phosphorylated proteins in mineralized tissues," Archives of Oral Biology, vol. 57, no. 9, pp. 1165-1175, 2012.

[49] B. Huang, I. Maciejewska, Y. Sun et al., "Identification of fulllength dentin matrix protein 1 in dentin and bone," Calcified Tissue International, vol. 82, no. 5, pp. 401-410, 2008.

[50] M.-G. Park, J.-S. Kim, S.-Y. Park et al., "MicroRNA-27 promotes the differentiation of odontoblastic cell by targeting APC and activating Wnt/ $\beta$-catenin signaling," Gene, vol. 538, no. 2, pp. 266-272, 2014.

[51] J. W. Kim, H. Choi, B. C. Jeong et al., "Transcriptional factor ATF6 is involved in odontoblastic differentiation," Journal of Dental Research, vol. 93, no. 5, pp. 483-489, 2014. 

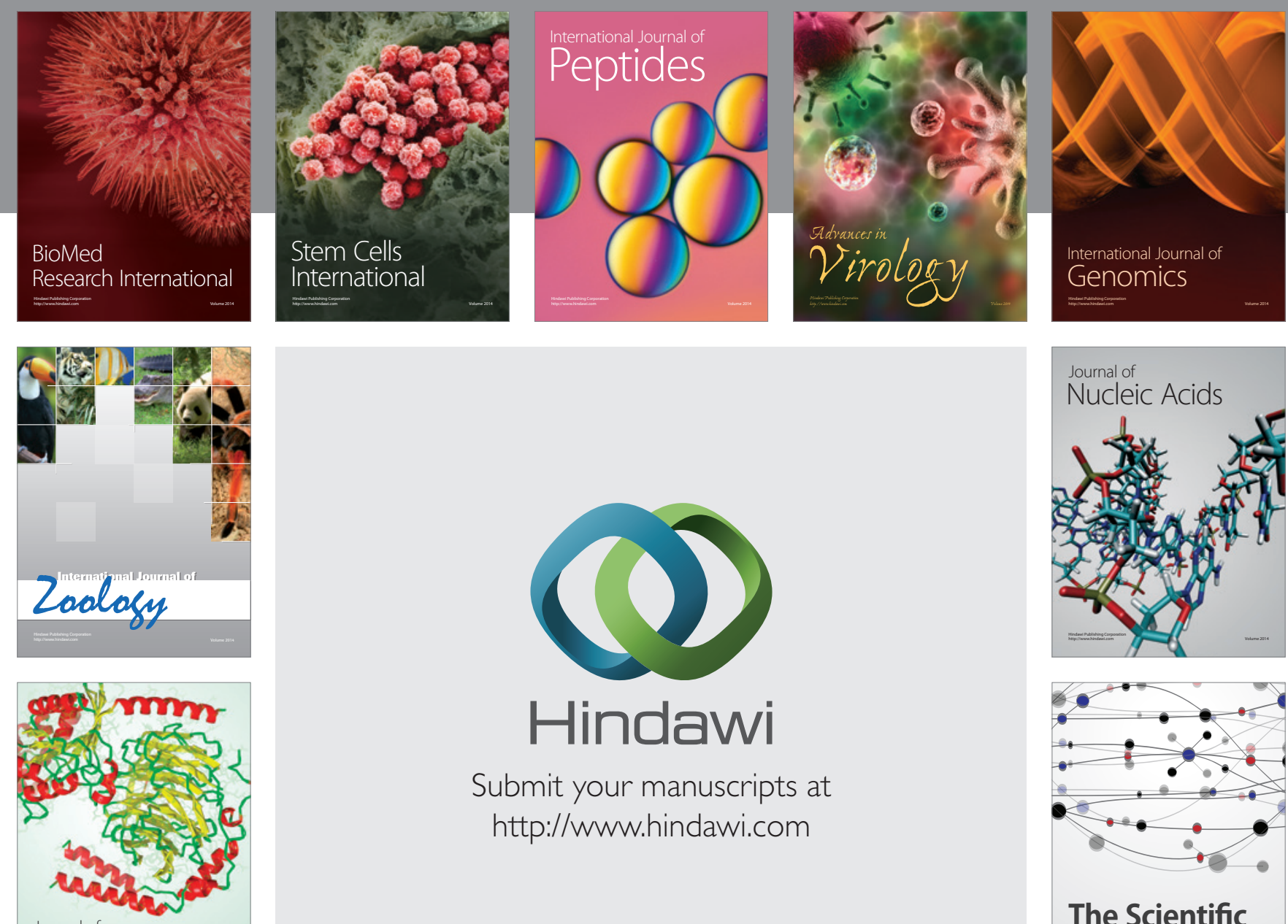

Submit your manuscripts at

http://www.hindawi.com

Journal of
Signal Transduction
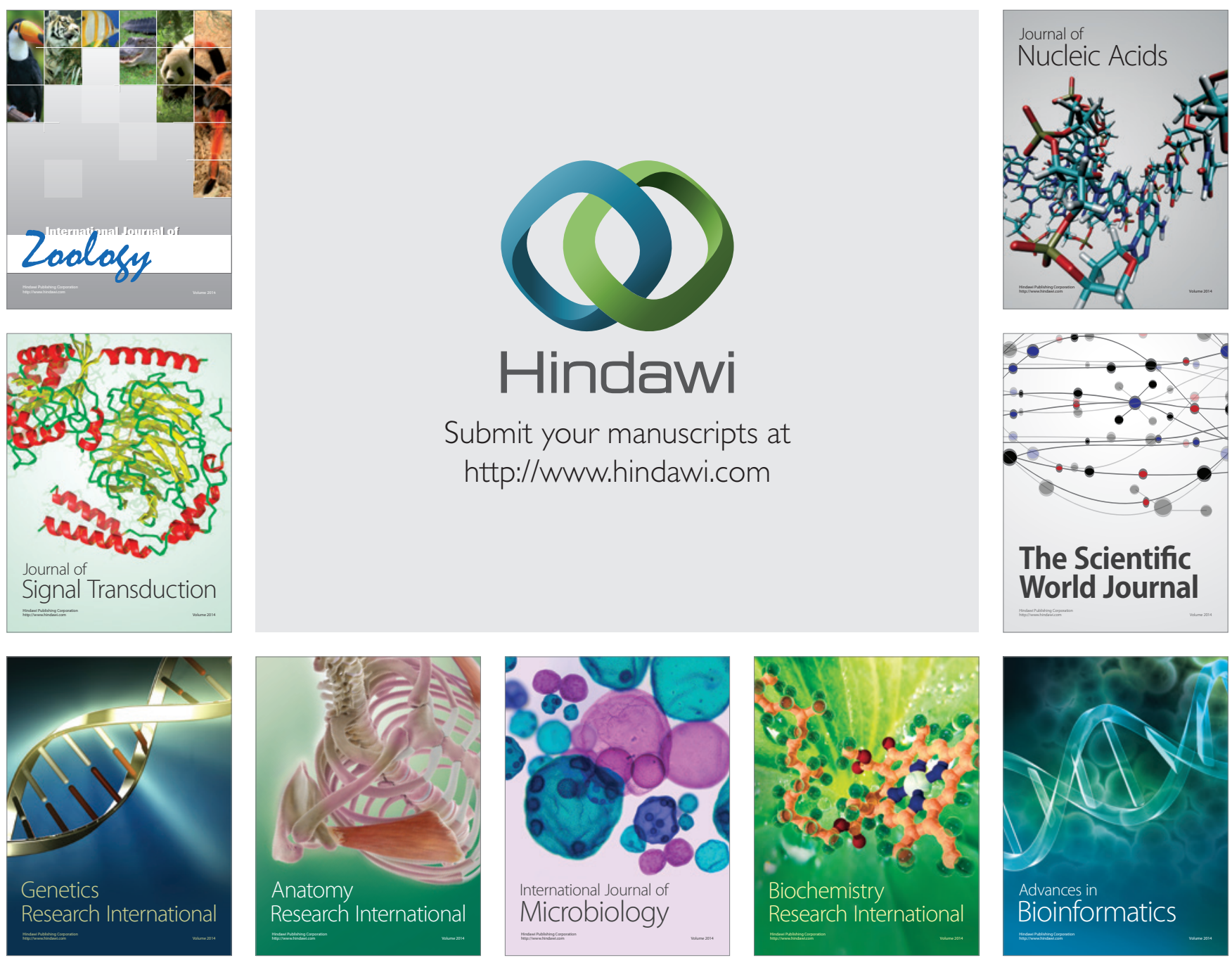

The Scientific World Journal
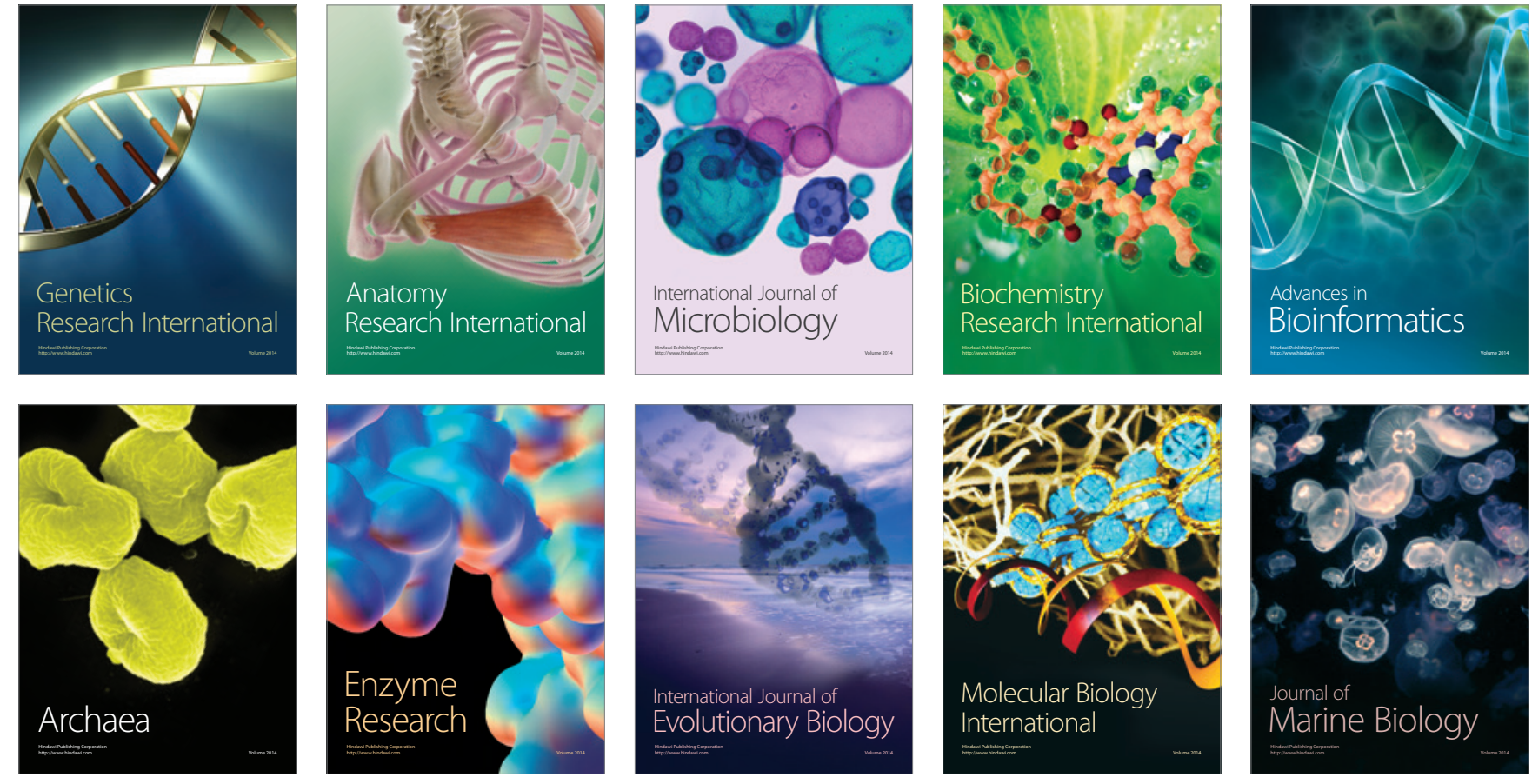